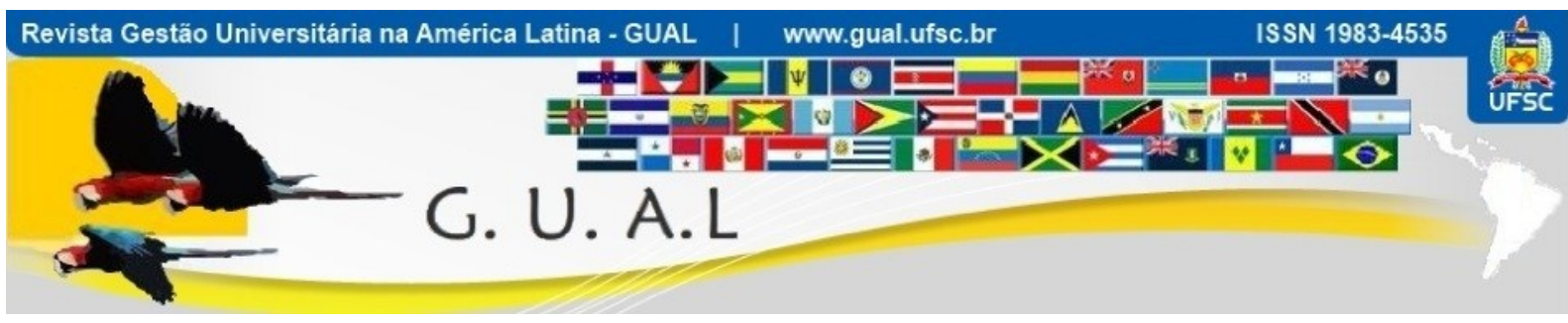

DOI: http://dx.doi.org/10.5007/1983-4535.2012v5n2p72

\title{
EL PROCESO DE PLANIFICACIÓN ESTRATÉGICA EN LAS UNIVERSIDADES: DESENCUENTROS Y RETOS PARA EL MEJORAMIENTO DE SU CALIDAD
}

THE PROCESS OF STRATEGIC PLANNING AT UNIVERSITIES: DISAGREEMENTS AND CHALLENGES FOR THE IMPROVEMENT OF HIS QUALITY

\author{
José Luis Almuiñas Rivero, Doctor \\ Universidad de La Habana/Cuba - UH \\ almu@,cepes.uh.cu \\ Judith Galarza López, Doctora \\ Universidad de La Habana/Cuba - UH \\ judith@cepes.uh.cu
}

Recebido em 25/maio/2012

Aprovado em 26/julho/2012

Sistema de Avaliação: Double Blind Review

Esta obra está sob uma Licença Creative Commons Atribuição-Uso. 


\title{
RESUMEN
}

En este artículo se presentan, inicialmente, algunos elementos teóricos-conceptuales relacionados con la dirección estratégica en el contexto universitario, haciéndose énfasis en uno de sus componentes principales: la planificación estratégica y el proceso que la sustenta. Posteriormente, se argumenta la necesidad de evaluar dicho proceso, como una vía para el mejoramiento de su calidad. El artículo tiene como objetivo identificar los principales problemas que están afectando la calidad del proceso de planificación estratégica en las universidades, así como proponer algunas direcciones de trabajo para su perfeccionamiento. Se han tomado como referencias diversas fuentes de información (asesorías, análisis documental y estudios empíricos desarrollados en varias universidades). Los resultados del análisis ponen en evidencia la presencia de diferentes tipos de problemas: conceptuales, metodológicos y técnicos, organizativos, culturales, entre otros. Como conclusión principal se señalan la importancia de realizar este tipo de evaluación en las universidades, con vistas a detectar a tiempo señales de aviso, que indiquen la presencia de insuficiencias y nuevas fortalezas existentes no descubiertas, para mantener en alto la calidad de dicho proceso y por tanto, mejorar la gestión institucional .

Palabras-claves: Dirección estratégica. Planificación estratégica. Proceso de planificación estratégica. Evaluación y calidad.

\begin{abstract}
In this article they encounter, initially, some theoretic conceptual elements related with the strategic management in the university context, becoming emphasis in one of his principal components: Strategic planning and the process that holds her. At a later time, process, like a road for the improvement of his quality arguments the need to evaluate saying itself. The article has like objective to identify the principal problems that are affecting the quality of the process of strategic planning at universities, as well as naming some directions of work for its perfecting. They have taken like various references sources of information (expert advices, documentary analysis and empiric studies developed at several universities). The results of analysis expose the presence of different kinds of problems: Conceptual, methodological and technicians, organizational, cultural, among others. As the importance to accomplish this type of evaluation at universities with an eye to detect signals of ad on time, that they indicate the presence of insufficiencies and new existent fortresses discovered, in order to bear up the quality of the aforementioned process indicate principal conclusion themselves and therefore, improving the institutional steps.
\end{abstract}

Key words: Strategic management. Strategic planning. Process of strategic planning. Evaluation and quality. 


\section{EL PROCESO DE PLANIFICACIÓN ESTRATÉGICA EN LAS UNIVERSIDADES: \\ DESENCUENTROS Y RETOS PARA EL MEJORAMIENTO DE SU CALIDAD \\ DOI: http://dx.doi.org/10.5007/1983-4535.2012v5n2p72}

\section{INTRODUCCIÓN}

Cada día el mundo se torna más inestable, turbulento e incierto, observándose que el cambio se ha convertido en una regularidad. Fenómenos que no existían hace unas pocas décadas, ya forman parte de los rasgos distintivos del panorama del siglo XXI, y ocasionan impactos negativos sin precedentes en todos los países. Esta realidad, acentuada en las naciones subdesarrolladas, es una permanente amenaza difícil de manejar ya que provoca rupturas, tanto en las relaciones de producción como en la superestructura, que han transformado el orden de las cosas y de las percepciones, cambian el modo de vivir, pensar y actuar de las personas y refuerzan el consumismo, la destrucción del medio ambiente, la agudización de las desigualdades y de la pobreza, entre otros males.

Ello obliga a las organizaciones, incluyendo las universidades, a reaccionar ante los riesgos y oportunidades con una nueva forma de sustentar su gestión. Uno de los enfoques, que ha ganado auge en las universidades latinoamericanas es la dirección estratégica, dentro de la cual se le está prestando especial atención a la planificación estratégica, como herramienta para orientar el rumbo institucional. La planificación estratégica se operacionaliza a través de un proceso, cuyo principal resultado es la Estrategia institucional. Mantener altos rendimientos en la gestión de dicho proceso exige desarrollarlo con calidad, y por tanto se necesitan acciones evaluativas, que propicien su mejoramiento continuo.

El objetivo de este artículo es poner de relieve la necesidad de evaluar dicho proceso cada cierto tiempo, para evitar que se vuelva rutinario. Se pretende además, identificar algunos problemas que afectan su desarrollo, tomando como referencia diversas fuentes de información. Finalmente, se presentan reflexiones, que permiten visualizar direcciones de trabajo orientadas a dar el salto de calidad que se necesita en el mismo.

\section{LA DIRECCIÓN ESTRATÉGICA EN EL CONTEXTO UNIVERSITARIO}

Ante el escenario internacional descrito, la educación superior en América Latina tiene importantes retos ${ }^{1}$ que enfrentar. En la Declaración de la Conferencia Regional de Educación Superior de América Latina y el Caribe (CRES 2008) se señala que:

Las Instituciones de Educación Superior deben avanzar en la configuración de una relación más activa con sus contextos (...). Ello exige impulsar un

\footnotetext{
${ }^{1}$ Se refiere a las exigencias que se derivan del entorno y del desarrollo de la educación superior y que determinan su orientación futura en términos de las prioridades principales.
} 


\section{EL PROCESO DE PLANIFICACIÓN ESTRATÉGICA EN LAS UNIVERSIDADES: DESENCUENTROS Y RETOS PARA EL MEJORAMIENTO DE SU CALIDAD DOI: http://dx.doi.org/10.5007/1983-4535.2012v5n2p72}

modelo académico caracterizado por la indagación de los problemas en sus contextos; una investigación científica, tecnológica, humanística y artística fundada en la definición explícita de problemas a atender, de solución fundamental para el desarrollo del país o la región, y el bienestar de la población; un trabajo de extensión que enriquezca la formación, colabore en detectar problemas para la agenda de investigación y cree espacios de acción conjunta con distintos actores sociales, especialmente los más postergados (IESALC-UNESCO, 2008, p. 7).

Pero ninguno de los retos mencionados podría lograrse, si se no potencia la gestión universitaria. Esta idea se reforzó también en la referida Declaración al plantearse:

Las Instituciones de Educación Superior de la región necesitan y merecen mejores formas de gobierno, capaces de responder a las transformaciones demandadas por los contextos internos y externos. Eso exige la profesionalización de los directivos y una vinculación clara entre la misión y los propósitos de la institución y los instrumentos de gestión (Ibíd., p. 5).

Las universidades tienen que operar en un medio de incertidumbre, turbulencia y complejidad, que les obliga a la búsqueda continua de la eficiencia y la eficacia de sus modelos de gestión. Y en este contexto, es un imperativo potenciar la planificación, y dentro de ella, su dimensión estratégica: la planificación estratégica.

En torno a la evolución de la Dirección, Administración o Gestión, resulta importante señalar su vinculación con el propio devenir histórico de la sociedad, teniendo su génesis en países como Egipto, China, Roma y Grecia. En la época medieval se destacaron los aportes de la Iglesia Católica, la Escuela Cameralista, el pensamiento de Maquiavelo, los escritos de Montesquieu, los Anales de la Revolución Francesa con la aparición del Estado de Derecho. La actividad militar también influyó en su desarrollo teórico. Sin embargo, el pensamiento más estructurado, científico, independiente y acelerado surge a partir de la Revolución Industrial, que posibilitó la creación de las primeras Escuelas de Administración.

En el siglo XIX surge la llamada Escuela Clásica, que tuvo dos componentes esenciales: la teoría de la Administración Científica y la teoría de las Organizaciones. Luego se desarrolló la Escuela de la Ciencia del Comportamiento y más adelante, las Escuelas de las Ciencias Administrativas y la Investigación de Operaciones. Posteriormente aparecen otros enfoques: el de sistema, el de contingencia y el nuevo movimiento de las relaciones humanas. Toda esta evolución constituyó el antecedente del surgimiento de la "Estrategia" y la dirección estratégica en el mundo empresarial y académico.

Existen muchas definiciones de "Estrategia" casi como autores han incursionado en el tema (Ansoff, 1976; Menguzzatto, 1984; Stoner, 1989; Steiner, 1991; Koontz, 1992; Porter, 1992). 


\section{EL PROCESO DE PLANIFICACIÓN ESTRATÉGICA EN LAS UNIVERSIDADES: DESENCUENTROS Y RETOS PARA EL MEJORAMIENTO DE SU CALIDAD DOI: http://dx.doi.org/10.5007/1983-4535.2012v5n2p72}

Para Ronda, algunos de los conceptos destacan el vínculo de la organización con el entorno, en otros se concibe como las vías para el cumplimiento de los objetivos y metas institucionales, o se refiere a la competencia entre las organizaciones (Ronda, 2007, p. 5).

Específicamente, el desarrollo de la dirección estratégica en el medio empresarial surge por el sobredimensionamiento de la aplicación de la planificación estratégica en las organizaciones, que será tratada más adelante. Al respecto, Menguzzatto y Renau plantearon:

La planificación estratégica aparece como un ataque solo parcial al problema estratégico. El análisis del entorno se basa en las variables económicas y tecnológicas obviando las variables psicosociopolíticas. La dinámica social y política, tanto dentro como fuera de la empresa, se supone irrelevante y sin ser afectada. Sin embargo, estas variables tienen y tendrán una importancia real cada vez mayor. Se pone el énfasis en la formulación de la Estrategia, suponiendo que la organización seguirá despreocupándose de las condiciones necesarias para la realización de la misma, así como de su control, cuando precisamente una mala ejecución puede significar el fracaso de la mejor Estrategia (Menguzzatto y Renau, 2000, p. 78).

La dirección estratégica se centra, tanto en las metas cuantitativas como cualitativas, contribuyendo en el logro de los propósitos socio-psicológicos de las organizaciones; ambas son importantes, en un mundo donde predominan los intangibles y las ventajas competitivas están más asociadas con el capital humano, con los conocimientos, con el aprendizaje, y sobre todo para las organizaciones de servicios y de valor intangible, donde se incluyen las universidades. Dicho enfoque de dirección se refiere a la toma de decisiones sobre los problemas más importantes que se presentan en una organización.

En las universidades, dicho enfoque ha ganado importancia. Al respecto, se plantea:

La dirección estratégica se convierte actualmente en una necesidad de las universidades, porque son organizaciones que poseen procesos conscientes muy complejos, interrelacionados entre sí y sujetos a un conjunto de exigencias muy dinámicas. A esta disyuntiva, debe dar respuesta la dirección estratégica orientada a la calidad. Ello requiere abrir procesos de reflexión sobre la naturaleza de las acciones, así como incluir en la agenda de las mismas orientaciones a mediano y largo plazo. Por tanto, la dirección estratégica significa concebir la universidad, mirando hacia el entorno, tomando como base un futuro factible a lograr (Almuiñas, 2001, s/p).

Dirigir estratégicamente una universidad significa: (a) concebir su desarrollo futuro tomando conciencia de las condiciones turbulentas que existen en el entorno, intentando evaluar los cambios que se producen en él y salir a su encuentro (sistema abierto); (b) priorizar los factores externos con relación a los internos, dando importancia también a los 


\section{EL PROCESO DE PLANIFICACIÓN ESTRATÉGICA EN LAS UNIVERSIDADES: \\ DESENCUENTROS Y RETOS PARA EL MEJORAMIENTO DE SU CALIDAD \\ DOI: http://dx.doi.org/10.5007/1983-4535.2012v5n2p72}

principales usuarios de los resultados institucionales y a los aliados estratégicos que puedan apoyar el cumplimiento de su misión; (c) asumir una actitud proactiva y emprendedora, combinando lo formal, la intuición y la creatividad, con un enfoque de futuro fundamentado y más realista; y (d), estar concientes de que no basta con diseñar la proyección estratégica institucional, sino que también es importante hacerla realidad a través de acciones que favorezcan el cambio o que enfrenten la resistencia interna, o sea se concibe como un sistema que integra la formulación, la implementación y el control de la Estrategia.

Un desempeño competente de los directivos universitarios en relación a este enfoque exige acciones claves: satisfacer, imaginar, visionar, crear, proyectar, priorizar, arriesgar, facultar, capacitar, actuar, cambiar, evaluar colectivamente y decidir con rapidez.

\section{LA PLANIFICACIÓN ESTRATÉGICA EN LAS UNIVERSIDADES Y EL PROCESO QUE LA SUSTENTA}

\subsection{Algunos fundamentos teóricos - conceptuales y características principales de la planificación estratégica en las universidades}

Específicamente, los inicios de la planificación de la educación superior en América Latina se ubican hacia finales de la década de los años 50 del pasado siglo, aunque con planes normativos incipientes, pocos estructurados y en forma de programación en ministerios, viceministerios, direcciones, departamentos estatales y en universidades. Ha tenido un largo período de ascenso, descenso y hasta de crisis. En una primera etapa primó la planificación tradicional, que fue muy criticada y que aún permanece como práctica en muchas universidades. En este caso se concibe la organización como un sistema cerrado, en el cual se elabora un plan articulado por unos pocos directivos; existe predominio de la centralización y el estilo de dirección autoritario; se extrapola el presente al futuro, entre otras características.

En la medida en que el entorno ha cambiado y los retos han crecido en complejidad, más necesaria se ha hecho la aplicación de la planificación estratégica en las universidades, considerada como un "sistema abierto", donde cada universidad está llamada a cambiar en la medida en que integra las diferentes informaciones provenientes del medio externo e interno.

Dentro de los enfoques de planificación más predominantes en el contexto universitario se encuentran los siguientes: (Almuiñas, 1999, p.16)

a) el enfoque basado en la estimación de la demanda social, que tiene su basamento principal en la previsión de la posible demanda estudiantil; 


\section{EL PROCESO DE PLANIFICACIÓN ESTRATÉGICA EN LAS UNIVERSIDADES: DESENCUENTROS Y RETOS PARA EL MEJORAMIENTO DE SU CALIDAD DOI: http://dx.doi.org/10.5007/1983-4535.2012v5n2p72}

b) el enfoque de recursos humanos - demanda económica - o matriz de insumo producto, que centra su atención en la determinación de los recursos humanos cantidad y tipo de profesional- que son necesarios formar;

c) el enfoque de la planificación normativa, basado en la conformación de una norma, en la que la educación superior es concebida como un "sistema cerrado". Esa norma se define como estado óptimo a alcanzar en el futuro determinado;

d) el enfoque de la planificación prospectiva, que se fundamenta en el diseño de los futuros posibles, para luego identificar el más factible, que sirve de marco de referencia a la acción en el presente. En este caso, el futuro se concibe, no solo como una tendencia continua de la historia -tendencias fuertes-, sino también como un resultado de la presencia de gérmenes de cambios, que pueden ser detectados en el presente y que impactan en el desarrollo de la educación superior, posibilitando posteriormente intervenir y reorientar el rumbo a través de la formulación e implementación de estrategias y acciones prioritarias;

e) el enfoque de la planificación estratégica, concebido como un proceso (conjunto de actividades y operaciones interrelacionadas entre sí, que producen un resultado sinérgico), que tiene como uno de sus objetivos básicos la formulación de la Estrategia institucional. Exige el ajuste continuo a las nuevas condiciones del entorno y contempla los posibles desarrollos futuros. Toma en cuenta variables, que la planificación tradicional no considera en todas sus magnitudes: variables políticas, los juegos de poder de los actores influyentes en la realidad social y los estudios de viabilidad, entre otras.

La planificación estratégica en una universidad se concibe como un proceso participativo, sistemático, crítico y autocrítico e integral, estructurado en varios momentos o fases, que permite formular, entre otros, objetivos y estrategias en diferentes horizontes de tiempo, que necesita información externa e interna, responde a las demandas del entorno y de la propia institución, y cuyos resultados requieren de seguimiento y evaluación (ob.cit., p.15).

A través de ella, se concibe la universidad orientada hacia el entorno, siguiendo su rumbo cambiante. Se centra en las decisiones prioritarias, no en los planes, los análisis, las previsiones y metas. Hace énfasis en el futuro institucional y en su construcción desde el presente. No es un esfuerzo por reemplazar la intuición y el juicio de los directivos, al contrario proporciona líneas de acción y directrices para tomar decisiones, complementando la creación y sabiduría de los directivos para pensar de modo innovador y actuar estratégicamente con el futuro en mente. Así se ha convertido en un proceso integrante de un sistema de dirección más amplio denominado "dirección estratégica".

Desde la segunda mitad de la década del noventa del pasado siglo y hasta la actualidad, la aplicación de la planificación estratégica en el contexto universitario ha tenido 


\section{EL PROCESO DE PLANIFICACIÓN ESTRATÉGICA EN LAS UNIVERSIDADES: DESENCUENTROS Y RETOS PARA EL MEJORAMIENTO DE SU CALIDAD DOI: http://dx.doi.org/10.5007/1983-4535.2012v5n2p72}

avances, pero también momentos de estancamiento. Por tanto, su desarrollo no siempre ha sido lineal y ascendente. Al respecto, Samoilovich señaló:

Las instituciones están mejorando sus prácticas de gobierno como resultado de los estímulos del contexto y por decisiones propias. Estas innovaciones están contribuyendo a una mejor gobernabilidad (...). La clave de la gobernabilidad radicaba en una planificación estratégica (...). Este no ha sido un proceso lineal, más bien ha habido procesos de encanto y desencanto con la planificación estratégica, ahora, ¿Por qué fracasan y han fracasado ejercicios de planificaron estratégica? La principal razón no es técnica (...). En una universidad la posibilidad de los directivos de afectar recursos y reasignar personal es muy limitada. Las universidades son entidades complejas, integradas por grupos de profesionales con una propia identidad y unidades académicas; cada una con su propia historia, programas de enseñanza a distintos niveles, etc. Cuanto mayor es su tamaño y mayor el espectro de disciplinas, mayor es su complejidad (Samoilovich, 2008, pp. 5, 21, 28).

Asimismo, por el alcance de sus resultados, la planificación estratégica puede ser clasificada, como un proceso estratégico ${ }^{2}$, que se estructura sobre determinadas demandas, con vistas a obtener los resultados (plan estratégico, desarrollo personal, imagen institucional, otros), a partir de determinados insumos o entradas (factor humano, aspectos tecnológicos, normativas, estructura, sistemas, otros), que son necesarios asegurar y que después se transforman paulatinamente, hasta alcanzar las salidas previstas (resultados e impactos).

En particular, el factor humano es esencial para lograr un proceso de planificación estratégica efectivo. Actualmente, este se ha convertido en el recurso estratégico más importante con que cuentan actualmente las universidades. Si se quieren obtener resultados sostenibles, hay que implicarlo directamente, convertirlo en objeto activo inseparable del proceso de planificación estratégica. En este caso, se incluyen: la participación, la motivación, la capacitación, la comunicación y el compromiso de los implicados, que de conjunto y en un marco de consenso, establecen la misión, la visión, los objetivos y las metas organizacionales y estrategias, tomando como base las demandas del entorno, así como las capacidades institucionales existentes. Todo ello implica además la necesidad de aplicar estilos y métodos de dirección proactivos, democráticos, una comunicación y motivación efectivas, la ampliación de conocimientos y habilidades para convertir dicho proceso en aprendizaje

\footnotetext{
${ }^{2}$ Aquellos procesos que establecen lineamientos, políticas y pautas generales de actuación para toda la organización, facilitan recursos que influyen directamente en su ejecución y tributan al análisis del funcionamiento del sistema establecido con el fin de proceder a su mejora continua.
} 


\section{EL PROCESO DE PLANIFICACIÓN ESTRATÉGICA EN LAS UNIVERSIDADES: DESENCUENTROS Y RETOS PARA EL MEJORAMIENTO DE SU CALIDAD DOI: http://dx.doi.org/10.5007/1983-4535.2012v5n2p72}

permanente e ir conformando así un pensamiento estratégico, que contribuya favorablemente en la cultura organizacional y en el desarrollo de un liderazgo colectivo.

En un grupo importante de instituciones y organismos regionales y nacionales de educación superior, la planificación estratégica ha avanzado en el diseño y aplicación de "modelos", que se han convertido en herramientas de trabajo que apoyan la gestión. Existen muchos modelos, que han sido diseñados y aplicados para conducir el proceso, tanto en el medio empresarial y como en el universitario, poniendo de relieve - según definen sus autores- los "momentos o fases" principales. Del análisis comparativo de un conjunto de propuestas, pueden señalarse algunos puntos comunes y diferencias:

- no existe un "modelo" único para diseñar la Estrategia en el medio empresarial y en el contexto universitario y los aportes en éste último, han sido menos significativos;

- una gran parte de los “modelos" analizados tiene más o menos similitud en algunos de sus momentos o fases, por ejemplo, la mayoría incluye el análisis del estado actual de la organización; se declara la misión y se formulan los objetivos y estrategias específicas (a corto, mediano y largo plazo); se evalúan los factores internos y externos, que influyen en la organización. Sin embargo, la secuencia de los momentos, así como los métodos y técnicas que emplean son muy diferentes y por tanto, no hay una regularidad notable. Esto es lógico si se tiene en cuenta que los supuestos que sustentan cada uno de los mismos no son homogéneos;

- no se resalta en todos el rol que tienen los implicados en el proceso (intereses de las personas y de otros actores), ni de los soportes que deben apoyar la ejecución y control de la Estrategia (estructura, presupuesto, normativas, sistemas, otros;

- existe pobre tratamiento a los valores compartidos y la visión; en la mayoría de los modelos, no se realiza el análisis de viabilidad de las estrategias formuladas;

- el momento de seguimiento y evaluación de la calidad del proceso no siempre se concibe como un factor esencial del mismo. (Almuiñas, 1999, p. 27).

Se puede plantear que en la literatura consultada por los autores de este artículo, no se encuentran muchos "modelos y metodologías" totalmente autóctonos en el contexto universitario, ya que una gran parte de los existentes se diseñan, aplican y perfeccionan, tomando como base aquellos que surgen del medio empresarial.

\subsection{La necesidad de evaluar la calidad del proceso de planificación estratégica en las universidades}

En los últimos años, las universidades han trabajado en función de elevar la calidad de los procesos que desarrollan. Ello ha estado vinculado con los cambios políticos, económicos 


\section{EL PROCESO DE PLANIFICACIÓN ESTRATÉGICA EN LAS UNIVERSIDADES: DESENCUENTROS Y RETOS PARA EL MEJORAMIENTO DE SU CALIDAD DOI: http://dx.doi.org/10.5007/1983-4535.2012v5n2p72}

y sociales que se han generado, obligándoles a aplicar, no en el discurso, sino en la práctica, los conceptos de eficiencia, eficacia, calidad y pertinencia en los procesos universitarios.

La calidad en la educación superior puede analizarse de múltiples y variadas formas. Emerge como un concepto relativo y multidimensional, que abarca el conjunto de las cualidades deseables en los resultados y en sus impactos, en los insumos a los procesos universitarios y en las condiciones internas con que estos se desarrollan. A su vez, considera la pertinencia, como el impacto de esos resultados a los propios fines institucionales, a los requerimientos de la ciencia en sí misma y a las necesidades de la sociedad.

Para Morales, la calidad en la educación superior contemporánea se basa en:

Una noción de cambio cualitativo, de transformación constante, utilizándose como un término de referencia de carácter comparativo, dentro de un conjunto de elementos homologables, a partir de cierto patrón o indicadores preestablecidos, siendo una resultante cualitativa de un conjunto de fuerzas impelentes, retardantes, estabilizadoras e impidientes, que tienen $\mathrm{su}$ origen en los diferentes grupos y posturas educativas que siempre coexisten en toda institución. Así, el mejoramiento de la calidad deja de ser un proceso lineal, para convertirse en un proceso omnidireccional y multifactorial, cuyos resultados van a diferir de acuerdo con los patrones de referencia empleados (Morales, 2009, p. 9).

Según De la Orden, existen tres imperativos esenciales de calidad que debe poseer un centro educativo: funcionalidad (se satisfacen necesidades del entorno), eficacia (alcanza los objetivos propuestos) y eficiencia (alcanza dichos objetivos con costo razonable) (2002, p. 3).

La calidad constituye también un objetivo central de la gestión universitaria y de los procesos que la integran. Hablar de calidad de la gestión, incluye también a la planificación estratégica y al proceso que la sustenta; significa lograr en el mismo progreso, transformación y un adecuado nivel de satisfacción de las demandas individuales, colectivas, institucionales, y en general, de la sociedad. Todo ello, apoyado con acciones evaluativas bien fundamentas.

La evaluación institucional en las universidades es un concepto ampliamente tratado en la literatura desde hace varias décadas y no deja de ser al mismo tiempo polémico, ya que no existe una concepción única por sus diversas connotaciones. Entre las definiciones más o menos comunes al respecto se señala la siguiente: "Proceso orientado a la toma de decisiones y a la acción, que busca determinar la pertinencia, la eficacia y el impacto del uso de recursos, actividades y resultados en función de objetivos pre-establecidos" (Martínez, 1997, p. 79).

Lo que si se ha observado es que los conceptos más recientes sobre la evaluación institucional están más vinculados a la problemática de la calidad como valor del mérito, la 


\section{EL PROCESO DE PLANIFICACIÓN ESTRATÉGICA EN LAS UNIVERSIDADES: DESENCUENTROS Y RETOS PARA EL MEJORAMIENTO DE SU CALIDAD DOI: http://dx.doi.org/10.5007/1983-4535.2012v5n2p72}

excelencia académica, la eficiencia, la pertinencia, el rendimiento, la responsabilidad social, entre otros. Los autores de este artículo, la conciben también como un proceso estratégico, continuo, integral y participativo, que mediante la obtención y análisis crítico de la información, permite medir, verificar, apreciar, calcular la situación de un objeto de estudio determinado, para emitir juicios de valor sobre aciertos y problemas fundamentales, a partir de los cuales se toman decisiones para elevar permanentemente la calidad institucional.

La evaluación institucional plantea y analiza la relación existente entre los objetivos previstos, los medios de los que se dispone para alcanzarlos y los resultados que finalmente se han obtenido. Su objetivo esencial es elevar la calidad de la institución a través de un plan de mejora. Cumple el propósito de rendir cuentas a la comunidad universitaria, a las instancias superiores y a la sociedad. Por tanto, las universidades, deben introducir la evaluación institucional con fines de autorregulación en su quehacer habitual, promoviendo la autoevaluación apoyada también con evaluaciones de pares externos, así como ser conscientes y aceptar la necesidad de evaluar sus procesos, ya que de ello depende la eficiencia y eficacia de sus resultados; es decir, el cumplimiento de su responsabilidad social.

Específicamente, cuando intentamos evaluar la calidad de la gestión en las universidades, un aspecto a considerar es el proceso de planificación estratégica, cuyos resultados tienen gran significación institucional. Los errores que se cometan en el mismo pueden influir negativamente en la calidad de la Estrategia institucional que se pretende elaborar, en los niveles de compromisos del factor humano y en otros resultados. Dicho proceso puede y debe ser revisado y perfeccionado periódicamente, para que se acomode a las cambiantes circunstancias externas e internas. Cualquier metodología que se desee aplicar, necesita de un tiempo para su avance y consolidación; son pocas las que han tenido éxito en un horizonte temporal relativamente corto; se requieren muchos esfuerzos y cambios culturales para su paulatina asimilación y comprensión por parte de los implicados.

Por ello, la evaluación de la calidad del proceso de planificación estratégica -en ocasiones ausente en muchas instituciones- no se puede concebir como un fin en si misma, sino es una vía para la mejora continua de una de las funciones básicas de la gestión universitaria. En esta evaluación se enfatiza en los aspectos conceptuales y metodológicos, en los procedimientos y normas de operación, así como con el factor humano.

$\mathrm{Su}$ importancia se ratifica cuando se señala: 


\section{EL PROCESO DE PLANIFICACIÓN ESTRATÉGICA EN LAS UNIVERSIDADES: DESENCUENTROS Y RETOS PARA EL MEJORAMIENTO DE SU CALIDAD \\ DOI: http://dx.doi.org/10.5007/1983-4535.2012v5n2p72}

Contar con una Estrategia bien fundamentada es una condición necesaria de la dirección estratégica para la calidad, es encontrar la orientación correcta de una universidad con relación a su entorno. No se puede pensar en la implementación y menos aún en el control, si no se diseña la Estrategia a través de un proceso de calidad para concebir y poner de relieve en ella, los elementos más importantes y prioritarios para el desarrollo futuro de la institución. Lo importante es iniciar y avanzar en los procesos de su implementación y control, para lograr la retroalimentación necesaria (Almuiñas, 2011, p. 5).

Al respecto, los problemas se pueden presentar en las tres etapas del proceso de planificación estratégica: (a) en su concepción inicial (planificación y organización del proceso); (b) su implementación o ejecución; y (c), en su seguimiento y control.

La evaluación de la calidad del proceso de planificación estratégica en una universidad debe estar concebida desde la propia etapa de su planificación y organización. Sin embargo, la práctica indica que no siempre esto se logra y muchas veces se ejecuta el mismo, sin concebir previamente, cómo se va a evaluar su calidad. Hay que partir del hecho que una cuestión es cómo queremos que se desarrolle el proceso de planificación estratégica (fundamentos teóricos-conceptuales - metodológicos) y otra es, su implementación y control en la práctica; ya que pueden ocurrir brechas entre lo previsto y lo real, lo oculto y lo vivido.

En este caso, a la universidad se le plantean un conjunto de aspectos interesantes a evaluar en dicho proceso, por ejemplo conocer si sus propósitos y resultados se lograron, como se ha desarrollado el contenido de los momentos que integran la metodología que se aplicó y si las condiciones para su aseguramiento garantizaron la calidad del mismo. Dicha evaluación debe abarcar también el factor humano que está implicado en el proceso.

Algunas reflexiones sobre las ideas expresadas anteriormente pueden brindar puntos de análisis diferentes sobre el tema en cuestión. Un proceso de planificación estratégica se puede diseñar para resolver determinados propósitos institucionales por ejemplo, mejorar el proceso de toma de decisiones, contribuir en la evaluación del desempeño institucional, apoyar la utilidad de una proyección estratégica, disminuir los gastos y mejorar la cultura organizacional. Si estas necesidades no están bien identificadas o están no resueltas, después de pasado un tiempo, es evidente que resulta conveniente producir cambios importantes en la gestión de dicho proceso; es decir, en su planificación, organización, ejecución y control.

Al mismo tiempo, el proceso debe orientarse a obtener determinados resultados que son parte integrante de la proyección estratégica y que deben poseer la calidad exigida en su formulación (misión, la visión, los objetivos y las estrategias específicas, etc.). También debe 


\section{EL PROCESO DE PLANIFICACIÓN ESTRATÉGICA EN LAS UNIVERSIDADES: \\ DESENCUENTROS Y RETOS PARA EL MEJORAMIENTO DE SU CALIDAD \\ DOI: http://dx.doi.org/10.5007/1983-4535.2012v5n2p72}

lograrse el mejoramiento de la calidad de la formación de los implicados, de la coordinación y comunicación externa e interna, del proceso de negociación y estimulación y, de otros aspectos vinculados con la cultura organizacional existente.

En resumen, la evaluación de la calidad del proceso de planificación estratégica debe ser un tema que se apoye más en la investigación, y convertirse también en parte de la agenda de la gestión universitaria actual. A veces una universidad opera durante varios años, con una misma concepción de este proceso, prestándole poca importancia a su perfeccionamiento. Es decir, se olvida del monitoreo de algunas señales que pueden dar luz para el cambio en el momento preciso. No verlo así, crearía fronteras innecesarias muy perjudiciales para las universidades, dejando que el mismo muera lentamente con los peligros de la burocratización y la rutina que puede llevar implícito y que no permiten el desarrollo creativo de los docentes, dirigentes y otros miembros de la comunidad universitaria, entorpeciendo además, cualquier iniciativa destinada a promover la cultura de planificación y de evaluación institucional.

\section{EL PROCESO DE PLANIFICACIÓN ESTRATÉGICA: UNA MIRADA CRÍTICA A PARTIR DE EXPERIENCIAS DESARROLLADAS EN UNIVERSIDADES LATINOAMERICANAS}

\subsection{Enfoque aplicado y fuentes de información para el análisis}

De la sistematización de las experiencias desarrolladas en varias universidades latinoamericanas y de los resultados de diversos estudios que los autores de este artículo han realizado en instituciones cubanas, resulta especialmente interesante la relación de problemas que están afectando la calidad del proceso de planificación estratégica.

Una primera instancia de información que fue utilizada para el referido análisis está vinculada con las labores de asesorías para conducir el proceso de planificación estratégica en diferentes universidades públicas y privadas (Cuba, Ecuador, Bolivia, México), que aportaron experiencias importantes en lo profesional y humano. Se debe señalar, que en cada caso fue evaluada la calidad de dicho proceso por los participantes, utilizando diversas técnicas.

En segundo lugar, hemos tomado como referencia, un conjunto de actividades académicas desarrolladas en cursos de superación profesional y en procesos de formación de másteres y doctores en la esfera de la gestión universitaria en instituciones de varios países (Cuba, Perú, Ecuador, México, Uruguay, Venezuela), donde el tema de la planificación estratégica siempre ha tenido un espacio propio. Ello nos ha permitido profundizar en otras 


\section{EL PROCESO DE PLANIFICACIÓN ESTRATÉGICA EN LAS UNIVERSIDADES: DESENCUENTROS Y RETOS PARA EL MEJORAMIENTO DE SU CALIDAD DOI: http://dx.doi.org/10.5007/1983-4535.2012v5n2p72}

experiencias institucionales e individuales, aportando visiones diferentes y enriqueciendo profundamente los análisis alrededor de la calidad del proceso que la sustenta.

Un tercer elemento a destacar en este punto es la valoración del contenido de los planes estratégicos de varias universidades, lo que nos aportó también informaciones de gran interés sobre dicho proceso y sus resultados.

Un último aspecto considerado son los resultados de estudios empíricos realizados en algunas universidades (Cuba, Bolivia, Ecuador), cuyo propósito central era precisamente, evaluar la calidad de dicho proceso a través de intervenciones directas. En este caso, el eje de la evaluación estuvo centrado, tanto en las características del proceso de planificación estratégica como en sus resultados, con énfasis en su impacto hacia los beneficiarios. Las variables e indicadores definidos tuvieron en cuenta cuatro núcleos fundamentales:

(a) los beneficios percibidos por los usuarios sobre los resultados que se han derivado de la ejecución y su alcance;

(b) el nivel de aseguramiento y de eficiencia de sus sistemas o requerimientos técnicos y las facilidades que los mismos deben aportar;

(c) los peligros latentes, que a criterios de los directivos, están presentes en el proceso;

(d) los errores reconocidos por los directivos en la operacionalización del proceso; y

(e) la identificación de las brechas existentes entre el estado deseado y la operacionalización real de algunas variables e indicadores que influyen en la calidad del proceso. Los nudos críticos de la evaluación se centraron en dos áreas: el factor tecnológico (aspectos metodológicos, organizativos técnicos, otros) y el factor humano (cómo las personas se insertan en el proceso).

Para el desarrollo de dichos estudios se tuvieron en cuenta, en algunos casos, los criterios de una muestra representativa de: directivos universitarios de primer nivel, intermedios y de base, profesores; trabajadores de apoyo y estudiantes. A tales efectos, se diseñaron cuestionarios y guías de entrevistas con el objetivo de conocer sus valoraciones sobre diferentes aspectos relevantes relacionados con la calidad del proceso de planificación estratégica. En otros, se convocaron talleres de discusión con la presencia de los implicados en el proceso para obtener información sobre las principales insuficiencias.

Integrando las perspectivas diferentes y comunes de los sujetos que participaron en dichos estudios, y a partir de la propia experiencia vivida por los autores de este artículo, se pueden plantear que existen actualmente un grupo de problemas, que están influyendo negativamente en la calidad del proceso de planificación estratégica en las universidades analizadas. Inclusive algunos de ellos, pudieran ser comunes a otras instituciones de la región. 


\section{EL PROCESO DE PLANIFICACIÓN ESTRATÉGICA EN LAS UNIVERSIDADES: DESENCUENTROS Y RETOS PARA EL MEJORAMIENTO DE SU CALIDAD DOI: http://dx.doi.org/10.5007/1983-4535.2012v5n2p72}

\subsection{Problemas identificados}

Como una primera aproximación, y dejando abierta las puertas, para plantear otros problemas, se han identificado nueve áreas, donde se concentran las mayores tensiones en el proceso de planificación estratégica en muchas universidades. Con ello, no se pretende tratar con profundidad cada uno de los mismos por razones de extensión de este artículo; solo serán presentados algunas de dichas áreas, para comenzar a demostrar la necesidad de evaluar la calidad del referido proceso por su repercusión en la orientación institucional futura. A saber:

- El entorno no tiene aún un rol relevante en el proceso de planificación estratégica.

El enfoque que prevalece en la gestión universitaria no es desde el entorno, sino más bien es hacia adentro. Las exigencias derivadas de algunos de los cambios del contexto externo que son previsibles, no se incorporan al proceso todo lo ágil que se necesita y por lo tanto, no se analizan con la profundidad requerida y con el tiempo suficiente sus impactos en las universidades. Este problema ha provocado también, que en ciertos momentos, exista un defasaje entre el contenido de su plan estratégico y las demandas del entorno.

Otro elemento a destacar está vinculado con el diseño de los posibles escenarios en que se va a mover la universidad, momento este de gran importancia dentro de la mayoría de las metodologías de planificación estratégica. Se reconoce que esta tarea es sumamente compleja y difícil, y más aún con la situación incierta y dinámica que se presenta en los contextos nacionales e internacional, donde existen fenómenos de diferentes tipos, que están impactando fuertemente el desarrollo futuro de las universidades. En la tecnología utilizada para diseñar dichos escenarios, las universidades no poseen mucha experiencia y por lo tanto, los mismos no han sido, hasta el momento, suficientemente fundamentados.

Por otra parte, existen problemas derivados de la insuficiente información que se dispone sobre el desarrollo del país y de cada región, lo que pudiera dar lugar también a la presencia de un determinado nivel de imprecisión en cuanto al contenido de los escenarios y sus posibles impactos en la Estrategia institucional. Tampoco se observa con frecuencia la participación de organizaciones importantes del entorno en los procesos de planificación estratégica que se desarrollan en las universidades.

- El modelo de cultura organizacional existente en las universidades aún no responde a las exigencias del proceso de planificación estratégica. 


\section{EL PROCESO DE PLANIFICACIÓN ESTRATÉGICA EN LAS UNIVERSIDADES: DESENCUENTROS Y RETOS PARA EL MEJORAMIENTO DE SU CALIDAD DOI: http://dx.doi.org/10.5007/1983-4535.2012v5n2p72}

Si bien se reconocen los avances e impactos favorables que ha tenido la planificación estratégica en muchas universidades, aún la cultura organizacional existente no responde a las demandas de este enfoque. En este caso, un punto a tratar son los valores compartidos organizacionales, los cuales, a nuestro juicio, no han jugado un papel relevante. Resolver esta problemática es una tarea compleja, que requiere mucho tiempo para su consolidación y es de gran importancia ya que constituye uno de los aspectos centrales para el desarrollo de la cultura estratégica organizacional. En la práctica, son pocas las experiencias que pudieran señalarse sobre la utilización de dichos valores como un medio de apoyo a la dirección estratégica universitaria.

Al respecto, se pueden plantear un conjunto de insuficiencias que están presentes en las universidades:

(a) el poco desarrollo de la tecnología empleada para el diseño, seguimiento y evaluación de los valores organizacionales;

(b) el proceso de su formulación ha sido breve, a veces formal e incompleto, sobre todo en los aspectos referidos a su conceptualización y las vías y mecanismos para su operacionalización posterior en la universidad;

(c) no se divulgan entre los miembros de la comunidad universitaria y por lo tanto, el personal no es consciente de los mismos;

(d) insuficiente nivel de preparación del personal universitario para comprender la importancia que tiene este aspecto en el desarrollo personal, colectivo e institucional; y

(f) se manifiestan estáticos y de forma homogénea en toda la universidad, sin que se tengan en cuenta otras exigencias y particularidades de los diferentes colectivos.

Por otra parte, en las universidades existen prácticas que limitan también el proceso de planificación estratégica, que se corresponden, quizás más con las tradiciones históricas en la forma de planificar y en general, dirigir las mismas. Por ejemplo, algunos directivos tienen trabas mentales en la instrumentación de un sistema de gestión relativamente nuevo para ellos, dando paso al pragmatismo en la concepción y forma de trabajo a través de la realización de procesos muy formales y poco fundamentados.

Ahora, hay otros aspectos que demuestran también que el estilo de dirección sigue siendo tradicional:

(a) no está consolidado aún el pensamiento estratégico en los directivos universitarios;

(b) predomina la dirección vertical e impositiva;

(c) no siempre se tiene claridad sobre lo importante y lo urgente, lo estratégico y lo operativo; 


\section{EL PROCESO DE PLANIFICACIÓN ESTRATÉGICA EN LAS UNIVERSIDADES: DESENCUENTROS Y RETOS PARA EL MEJORAMIENTO DE SU CALIDAD DOI: http://dx.doi.org/10.5007/1983-4535.2012v5n2p72}

(d) es insuficiente el trabajo en equipo;

(e) existen dificultades en la comunicación vertical y horizontal, que afectan la integración real del plan estratégico y sus resultados entre los niveles de dirección;

(f) el control de los objetivos y metas es insuficiente; y

(g) no siempre se utiliza la proyección estratégica, como un instrumento de apoyo a la toma de decisiones, y cuando se hace, es muy formal, sin actuar consecuentemente con lo planeado.

Estos ejemplos son suficientes para poner de manifiesto algunas insuficiencias que están incidiendo negativamente en la cultura del cambio que exige la planificación estratégica y en el nivel de credibilidad existente sobre sus ventajas, una vez que se pone en práctica.

- Insuficiente participación de los implicados en el proceso de planificación estratégica y en la evaluación de sus resultados.

Aunque pudieran presentarse excepciones, parece que en la mayoría de las universidades estudiadas y posiblemente en otras más, no se han diseñado espacios de participación suficientes para integrar adecuadamente todos los miembros de la comunidad alrededor del proceso. De no mejorarse esta situación, se seguirían afectando los niveles de implicación y de credibilidad en la planificación estratégica, así como se engendraría paulatinamente un determinado nivel de autoritarismo, resistencia al cambio, desmotivación y de desinformación en una parte de la comunidad universitaria.

- El nivel de preparación de los docentes, trabajadores de apoyo, estudiantes y algunos directivos de los niveles intermedios y de base es insuficiente para asimilar adecuadamente las exigencias de la planificación estratégica.

Hasta el momento, los esfuerzos orientados hacia la capacitación del personal se han concentrado más bien en las altas autoridades, donde el nivel de formación es mayor que en la base. La mayor parte de la comunidad universitaria no conoce suficientemente los presupuestos generales de este enfoque, sus beneficios y exigencias principales, así cómo su verdadero rol en el proceso. Este problema incluye el dominio de técnicas, las cuales no siempre han sido correctamente aplicadas o su uso es aún pobre en algunas universidades. Por otra parte, parece aún insuficiente también el intercambio de experiencias, tanto al interior de cada universidad como entre las propias instituciones universitarias.

- La divulgación sobre los aspectos relacionados con la planificación estratégica hacia la comunidad universitaria es aún insuficiente. 


\section{EL PROCESO DE PLANIFICACIÓN ESTRATÉGICA EN LAS UNIVERSIDADES: \\ DESENCUENTROS Y RETOS PARA EL MEJORAMIENTO DE SU CALIDAD \\ DOI: http://dx.doi.org/10.5007/1983-4535.2012v5n2p72}

Todavía una parte de la comunidad universitaria no posee la información mínima necesaria sobre aspectos relevantes que tienen que ver con dicho proceso y sus resultados. Tal es el caso del poco nivel de conocimiento que declara tener una parte de los miembros de la universidad sobre la misión, la visión, los objetivos y metas formuladas anualmente. También existen problemas con el nivel de información que tienen sobre los resultados de la implementación del plan estratégico en los ámbitos institucional y facultativo.

- El proceso de planificación estratégica se realiza en un margen de tiempo muy inferior al necesario.

Al proceso de planificación estratégica no se les dedica un tiempo adecuado para reflexionar y profundizar en un grupo de aspectos, que son necesarios articular bien y que poseen gran trascendencia para el desarrollo institucional (misión, visión, escenarios, metas, etc.). A veces se acelera mucho el proceso por falta de tiempo, se precipitan algunos pasos y se realiza con mucho nivel de formalidad en períodos muy cortos y con poco apoyo de una tecnología básica. Todo ello está provocando que exista un determinado nivel de empirismo en las propuestas iniciales de la proyección estratégica, con efectos negativos en la ejecución $\mathrm{y}$ en los resultados que se alcanzan posteriormente.

- No siempre los recursos económicos - financieros disponibles son balanceados con la Estrategia diseñada.

Una de las insuficiencias existentes es que aún no se balancean previamente los recursos económicos - financieros disponibles para respaldar las prioridades especificadas en la Estrategia, es decir, está ausente el análisis de la viabilidad de la propuesta, lo que ha traído como consecuencia incumplimientos no asociados a problemas en su gestión. Aún cuando se quiera, a veces la disponibilidad de recursos no siempre alcanza para garantizar el cumplimiento de todas las metas, constatándose que estas son muy tensionantes en términos económicos - financieros. Por otra parte, se plantea la dificultad de la desvinculación entre la actividad académica y la actividad económica, lo que en la práctica es reflejo de lo expresado anteriormente. Quizás, en ambas áreas existen dos culturas diferentes, tanto en la forma de proyectarse para formular los objetivos institucionales como en su operacionalización. 


\section{EL PROCESO DE PLANIFICACIÓN ESTRATÉGICA EN LAS UNIVERSIDADES: \\ DESENCUENTROS Y RETOS PARA EL MEJORAMIENTO DE SU CALIDAD \\ DOI: http://dx.doi.org/10.5007/1983-4535.2012v5n2p72}

- No se dispone de un sistema de información que apoye las decisiones fundamentales para llevar a cabo el proceso de planificación estratégica en las universidades.

Un aspecto importante que se deriva del estudio realizado es que aún el sistema de información existente no responde a las exigencias básicas del proceso. Esto ha ocasionado que en determinados momentos -previo al inicio o durante el proceso- ha faltado información estratégica, generándose un cierto nivel de improvisación en algunas de sus etapas (formulación de los escenarios, la visión, las metas y la evaluación de su cumplimento).

- Las investigaciones relacionadas con la calidad del proceso de planificación estratégica son insuficientes.

En la actualidad existen pocas investigaciones y estudios que apunten hacia el mejoramiento de dicho proceso en las universidades y por lo tanto, las posibilidades reales de su perfeccionamiento se ven actualmente en algo limitadas. Este factor, unido a la poca experiencia e insuficiente literatura especializada en dicha área, exige una mayor respuesta dentro de los planes de investigación. Una gran cantidad de problemas que se presentan en dicho proceso, crean carpetas que pasan mucho tiempo sin ser investigadas, porque no tienen suficiente desarrollo en el contexto universitario. Por eso, la reflexión en torno a este aspecto conduce también hacia la necesidad de tener bien definidas algunas prioridades, donde bien vale la pena que existan proyectos de investigación.

\subsection{Hacia el mejoramiento del proceso de la planificación estratégica en las universidades de América Latina}

Muchas metodologías ya probadas en la práctica de la planificación estratégica en el contexto universitario se han convertido en pasivas y por tanto, requieren que se les añada valor, ante un entorno que seguirá cambiando constantemente. Construir una estructura más innovadora para fundamentar mejor el proceso, en lo teórico y metodológico, es uno de los retos más importantes que enfrenta la dirección estratégica universitaria. Entonces ¿Cómo podrán concretamente las universidades dar el salto que tanto se necesita?

Siete aspectos parecen integrar, en la perspectiva, la estructura de un pensamiento que pudiera valorizar más el proceso de planificación estratégica y por tanto, no deben ser obviados, tanto en la concepción y el diseño de un modelo teórico y de una metodología para su implementación (Almuiñas, 2009, 15). 


\section{EL PROCESO DE PLANIFICACIÓN ESTRATÉGICA EN LAS UNIVERSIDADES: DESENCUENTROS Y RETOS PARA EL MEJORAMIENTO DE SU CALIDAD DOI: http://dx.doi.org/10.5007/1983-4535.2012v5n2p72}

Un primer aspecto está vinculado con el logro de una adecuada combinación entre el tiempo que se dedica al diseño de la Estrategia y la velocidad para su aplicación.

El análisis y la reflexión que se deriva del diseño de la Estrategia son muy necesarios, pero no se llegará a ningún lugar, sino se aplica la misma rápido. El alto dinamismo del entorno exige rapidez en la toma de decisiones, en la delegación de autoridad, en la elaboración de los planes tácticos y operativos, que contengan las acciones y tareas que cada nivel de dirección (organismo nacional, instituciones, facultad, direcciones, departamentos o cátedras, otros) debe asumir y cumplir en la descentralización de la proyección resultante.

Otro de los problemas que se presenta comúnmente es que se realiza el diseño de la Estrategia, sin la participación de los niveles de dirección inferiores y en general de los trabajadores, por tanto, estos están ajenos a ella, sin compromiso con su cumplimiento, se pierde por tanto, el enfoque de sistema.

La Estrategia diseñada debe llegar a toda la organización, ser comunicada y explicada, para que se comprenda y se aplique; o sea, estar cerca de los que la ejecutan. Este proceso de derivación de la Estrategia debe ser integrado, de manera equilibrada y coordinada, donde cada una de las personas tribute a los fines globales de la universidad, en interrelación e interdependencia con todas las estructuras. En resumen, tiene que convertirse en un eje importante del sistema de dirección estratégica, que movilice y sirva guía en los niveles de dirección. Si la Estrategia no se implementa rápidamente, el proceso se convierte en un ejercicio teórico, en un sueño irrealizable y puede generar desmotivación y falta de compromisos de las personas. Por tanto, es peor el impacto de no aplicarla, que no formularla.

Un segundo aspecto que parece importante tiene que ver con la promoción de innovaciones paulatinas en el proceso de planificación estratégica.

Como expresamos anteriormente, mantener el mismo sustento teórico y metodológico durante mucho tiempo parece que no es una buena opción, al menos eso manifiestan muchas experiencias, que se convierten en rutinarias para los implicados.

Las mejores prácticas en el proceso de planificación estratégica se presentan en aquellas universidades, que cambian y aprenden constantemente, lo cual le agregaría un nuevo valor también a la calidad del mismo. Entonces, vale la pena destacar la necesidad de evaluar críticamente nuestros propios errores y aprender de ellos y de los logros alcanzados. Esto ratifica la necesidad de dar un espacio para la evaluación del proceso, una vez que se culmine. El otro extremo sería caer en el "más y más de lo mismo", con actitudes mecanicistas y estilos 


\section{EL PROCESO DE PLANIFICACIÓN ESTRATÉGICA EN LAS UNIVERSIDADES: DESENCUENTROS Y RETOS PARA EL MEJORAMIENTO DE SU CALIDAD DOI: http://dx.doi.org/10.5007/1983-4535.2012v5n2p72}

tradicionales de comportamiento para desarrollarlo. Esta inercia parece que no es una buena opción para aquellas universidades, que desean mejorar constantemente la dirección estratégica. En este contexto, vale la pena plantear también la necesidad de lograr un verdadero balance entre el proceso formal para diseñar la Estrategia y la capacidad de intuición de los directivos universitarios, lo que posibilita incorporarle determinadas dosis de innovación a su implementación y realizar adaptaciones continuas a las nuevas condiciones.

Un tercer punto está vinculado con el tratamiento que se da al futuro como un referente en el proceso de planificación estratégica.

En las universidades no se puede entender la planificación estratégica si no se concibe proactivamente el futuro factible de alcanzar en su dinámica para construirlo con acciones desde el presente. La planificación tradicional es por el contrario determinista y lineal, le da mucho valor al pasado y al presente para proyectar el futuro; es por tanto, reactiva. Para la planificación estratégica la construcción de diferentes futuros posibles y la selección dentro de ellos, del más factible, se convierte en un referente importante en el diseño de la Estrategia. En este caso, adquiere valor la Prospectiva, cono disciplina científica, con sus correspondientes modelos, metodologías y caja de herramientas. En esta dirección aún existe poco desarrollo en las universidades, a pesar de que su campo de utilización es muy amplio.

Un cuarto elemento a destacar tiene que ver justamente con la concientización del fuerte nexo entre el entorno y las bases en la que descansa la Estrategia y sus resultados.

En este caso se produce la relación desafío - respuesta; el primero surge del entorno, la segunda de la universidad y así la fórmula se repite continuamente. La universidad debe asumir una actitud preconcebida más abierta, crítica y extrovertida hacia el entorno; es decir mantener una interrelación constante con su contexto. Fortalecer dicha integración, transita por crear y utilizar diversas vías y medios, que permitan obtener informaciones útiles sobre actores y organizaciones claves del entorno (aliados estratégicos), así como de los usuarios de los resultados (empleadores y otras entidades del mundo laboral y científico), entre otros.

Hasta tanto no se logre un buen posicionamiento de las universidades ante los cambios previsibles del entorno, la planificación estratégica no se convertirá en cualidad del quehacer universitario. Visto desde esta perspectiva, las mismas requieren una actitud más activa en cuanto a la realización de estudios específicos sobre el contexto externo, que permitan identificar, de forma aproximada, las variables o tendencias pesadas de mayor influencia para su desarrollo futuro. Necesariamente habría que trabajar también en los aspectos tecnológicos 


\section{EL PROCESO DE PLANIFICACIÓN ESTRATÉGICA EN LAS UNIVERSIDADES: DESENCUENTROS Y RETOS PARA EL MEJORAMIENTO DE SU CALIDAD DOI: http://dx.doi.org/10.5007/1983-4535.2012v5n2p72}

para el diseño de los posibles escenarios, donde la metodología prospectiva pudiera brindar un espacio para la reflexión.

Otro de los elementos que requiere la atención está ligado al hecho de la necesidad de mantener lo más actualizado la proyección estratégica. Parece aconsejable entonces que todos los años se proceda a reajustar -si corresponde- los componentes principales de la proyección institucional; se incorporen los nuevos retos e inclusive, prescindir de otros si las circunstancias lo ameritan, sobre todo por razones económicas. Esto le pudiera dar un mayor dinamismo a la planificación estrategia en cada universidad.

Como quinto aspecto aparece la necesidad de alinear los tres componentes o funciones que integran la dirección estratégica universitaria.

Se ha señalado anteriormente que una de las críticas más comunes a la planificación estratégica es que no siempre una buena formulación implica necesariamente una correcta implementación y control ya que con frecuencia diseños bien formulados, no logran llevarse a la práctica. El problema principal es que a veces dichos procesos transcurren de manera dicotómica, uno separado del otro, o no logran concebirse como fases integradas. Se formula la Estrategia y luego se trata de ejecutar, cuando en la realidad el aseguramiento inicial de su implementación debe formar parte también del diseño estratégico, como también debe ocurrir con el control. Lo cierto es que hoy se ha avanzado mucho más en el dominio teórico y en las habilidades para la formulación de la Estrategia que en su implementación y control. Por tanto, el proceso de planificación estratégica tiene que resolver la contradicción que se ha dado entre la formulación, la implementación y el control, concibiendo estos dos últimos como partes inseparables de la primera.

De ahí, surge la necesidad de pensar -desde el momento de diseño de la Estrategia- en la formulación de soportes estratégicos (sistemas de información, comunicación, seguimiento y control de los objetivos, control estratégico, cuadro de mando integral, otros). Al respecto, diferentes autores señalan algunos que contribuyen en la implementación de la Estrategia. Menguzzato y Renau plantean los siguientes: la organización y estructura organizacional, la dirección y el liderazgo, la cultura organizacional, los sistemas de planificación y control, el sistema de información y el sistema de comunicación (2000, p. 295). Waterman, Peters y Philips, citados por Rodríguez y Alemañy incluyen como soportes: la estructura, los sistemas, el estilo de dirección, las habilidades, el personal y los valores compartidos (1998, p. 36). 


\section{EL PROCESO DE PLANIFICACIÓN ESTRATÉGICA EN LAS UNIVERSIDADES: DESENCUENTROS Y RETOS PARA EL MEJORAMIENTO DE SU CALIDAD DOI: http://dx.doi.org/10.5007/1983-4535.2012v5n2p72}

El sexto aspecto está vinculado con la necesidad de potenciar la cultura estratégica, para que responda mejor a las exigencias del proceso de gestión estratégica implantado en las universidades. Muchos de los problemas detectados están exigiendo un cambio cultural, de creencias, de valores, de actitudes y hábitos de la comunidad universitaria. Sobran los elementos para demostrar que el modelo cultural que hoy le interesaría desarrollar a las universidades, rompe con el esquema de los viejos hábitos en la gestión tradicional, por lo que en este sentido, debe existir una buena disposición al cambio en las universidades.

Pero no se trata de cambiarlo todo, sino solamente aquellos aspectos de interés para la Estrategia de la universidad. Esto exige un análisis inicial sobre lo que la universidad entiende que debe cambiarse, profundizando en cómo se hacen actualmente los diferentes procesos, cómo deberían llevarse a cabo los mismos, cuáles son las limitantes principales, cuáles son los valores organizacionales actuales y cuáles deberían ser en el futuro. Se deben definir cuales son los principales rasgos de la cultura actual y cuál es la que prefiere cada universidad de acuerdo a sus características y demandas particulares. Esta es una de las posibles dimensiones de un problema muy complejo que está aún por resolver.

En el centro de estas cuestiones se encuentra la realización de un trabajo más profundo alrededor de los valores compartidos de la universidad como expresión de unidad, cohesión y pertenencia a la organización. Un requisito imprescindible para tratar de modificar comportamientos y hábitos de las personas que integran la comunidad universitaria es que los directivos, en todos los niveles, en primera instancia, tengan una adecuada actuación en correspondencia con los valores compartidos declarados en la proyección estratégica de la universidad; es decir, actúen con autoridad moral y ejemplo ante los demás.

El séptimo aspecto está vinculado con la necesidad de generar modelos y metodologías propias, y no copiar aquellos que se aplican en el medio empresarial.

Este planteamiento ha sido muchas veces polémico. Aunque la planificación estratégica tuvo sus orígenes en la empresa, ella es válida y aplicable en cualquier organización, incluyendo a las universidades. Sin embargo, en estas últimas debe implementarse como un proceso de adaptación, de particularización y de singularización. Por tal motivo, dichas instituciones no deben copiar textualmente los modelos y metodologías de planificación estratégica que han sido exitosas en las empresas; su diseño debe ser un traje a la medida, ajustado a las condiciones concretas, a su prospección, al cambio necesario, a la actitud de los directivos y líderes universitarios, a la cultura organizacional. Se parte del principio de que las 


\section{EL PROCESO DE PLANIFICACIÓN ESTRATÉGICA EN LAS UNIVERSIDADES: DESENCUENTROS Y RETOS PARA EL MEJORAMIENTO DE SU CALIDAD DOI: http://dx.doi.org/10.5007/1983-4535.2012v5n2p72}

universidades no son empresas; sus misiones, objetivos y formas de tomar decisiones son distintas, al igual que la naturaleza y complejidad de los procesos que desarrollan. No obstante, son organizaciones, a las cuales mucho les aporta la planificación estratégica, como herramienta que direcciona el cambio y perfecciona su gestión. El otro extremo puede ser aceptar todo lo contrario y enfrentar las fuertes contradicciones que se generan.

Parece oportuno además proponer otras acciones:

(a) crear más espacios de participación para los estudiantes, docentes y otro tipo de personal;

(b) incrementar la preparación de la comunidad universitaria;

(c) estimular el intercambio de experiencias institucionales;

(d) potenciar la vinculación del área académica y el área económica-financiera;

(e) operacionalizar la Estrategia a través de planes tácticos y operativos;

(f) apoyar la gestión del proceso con las TIC;

(g) mejorar la planificación del tiempo para ejecutar el proceso; y

(h) lograr una mayor correspondencia entre la Estrategia y los estándares de calidad establecidos en el proceso de evaluación institucional con fines de autorregulación.

Entonces, se podría pensar que todo lo planteado anteriormente, conduce a introducir algunos cambios en la forma de concebir teórica y metodológicamente el proceso de planificación estratégica y su gestión en las universidades. Lo que se está discutiendo en estos momentos son alternativas, que viabilicen dicho proceso y por ello, las universidades deben ayudar, colaborar, participar y ser partes integrantes de ese cambio necesario en la concepción y diseño de modelos teóricos y metodologías propias que necesitan, para contribuir paulatinamente en el mejoramiento de la gestión del proceso de planificación estratégica.

Esto caminos no son los únicos posibles, quizás algunos no son viables y pueden encontrarse otros más válidos. Con el análisis realizado, solo se ha pretendido motivar la reflexión de los directivos y especialistas para tratar de llegar a consensos significativos sobre la mejor forma de elevar la calidad de la planificación estratégica en las universidades. No es relativamente reciente el abordaje de este tema, que tanto en la práctica como en la teoría, se está produciendo, pero que está lejos de resolverse; a lo sumo, podría decirse que tiene un retraso con respecto al medio empresarial. Lo cierto es que en los últimos años, las cuestiones relacionadas con el diseño, implementación y control de la Estrategia, son áreas de conocimientos, que se han puesto en el orden del día en muchos contextos universitarios. 


\section{EL PROCESO DE PLANIFICACIÓN ESTRATÉGICA EN LAS UNIVERSIDADES: \\ DESENCUENTROS Y RETOS PARA EL MEJORAMIENTO DE SU CALIDAD \\ DOI: http://dx.doi.org/10.5007/1983-4535.2012v5n2p72}

\section{CONCLUSIONES}

La aparición de nuevos agentes de cambio en un entorno dinámico y turbulento en los ámbitos regional, nacional e internacional exige la aplicación de enfoques de gestión cada vez más eficientes y eficaces en las universidades para que contribuyan a dar una respuesta favorable a las demandas que surgen en tales circunstancias. En este contexto, un aspecto de gran importancia es el mejoramiento de la planificación estratégica, que propicie al mismo tiempo elevar la calidad de los procesos internos y sus resultados e impactos en el entorno.

La consolidación de la planificación estratégica es un proceso largo y requiere de mucho esfuerzo por las universidades, en particular de sus directivos. Es así, que desde el punto de vista académico y científico, este artículo presenta interés, pues trata más que, identificar problemas, desarrollar un pensamiento colectivo, que consolide la idea sobre la necesidad de evaluar cada cierto tiempo, el proceso de planificación estratégica, lo que debe convertirse en una práctica actual, que forme parte del mejoramiento institucional. A través de dicha evaluación, se pudieran detectar a tiempo señales de aviso, que pongan de relieve la presencia de insuficiencias y nuevas fortalezas existentes no descubiertas aún, y manifiesten la necesidad de cambios en la gestión de dicho proceso. Son relativamente recientes los esfuerzos que se están realizando en esa dirección. Seguramente, los puntos de desencuentros identificados y otros no tratados, se manifiestan de forma diferenciada en las universidades, y están lejos de convertirse en un situación grave, pero si requieren de atención inmediata.

Finalmente, las investigaciones en la esfera de la dirección estratégica universitaria no han tenido el desarrollo suficiente que la situación actual hubiera requerido. Por un lado, no llegan aún a integrar un cuerpo sistemático de conocimientos, apoyado en las prácticas de dirección exitosas y por otro, existen pocos grupos de investigación consolidados e interrelacionados que permitan avances significativos. Su desarrollo teórico, metodológico e instrumental debe constituir una tarea de primer orden para las universidades, convirtiéndose en una necesidad actual para el perfeccionamiento de su sistema de gestión vigente.

\section{REFERENCIAS}

ALMUIÑAS, José L. (1999). La planificación estratégica en las Instituciones de Educación Superior. Tesis de doctorado en Ciencias de la Educación. Universidad de La Habana. Cuba.

ALMUIÑAS, José L. (2001). Introducción a la planificación y dirección estratégica en la educación superior. Material expositivo e impreso. En: Maestría en Ciencias de la Educación Superior. Escuela Latinoamericana de Medicina. La Habana. Cuba. 


\section{EL PROCESO DE PLANIFICACIÓN ESTRATÉGICA EN LAS UNIVERSIDADES: DESENCUENTROS Y RETOS PARA EL MEJORAMIENTO DE SU CALIDAD DOI: http://dx.doi.org/10.5007/1983-4535.2012v5n2p72}

ALMUIÑAS, José L. (2009). Dirección estratégica en las IES. Material expositivo e impreso. En: Maestría en Ciencias de la Educación Superior. Centro de Estudios para el Perfeccionamiento de la Educación Superior. Universidad de La Habana. La Habana, Cuba.

ALMUIÑAS, José L.; GALARZA, J. (2011). Hacia la búsqueda de nuevos modelos y metodologías de planificación estratégica en las Instituciones de Educación Superior. En: Coordinación General de RED-DEES (Orgs.). "Planificación Estratégica en las Instituciones de Educación Superior". Editora Eduquil. Universidad Estatal de Guayaquil. Ecuador (aprobado para su publicación).

DE LA ORDEN, A. (2002). Desarrollo y validación de un modelo de calidad universitaria como base para su evaluación. Revista Electrónica de Investigación y Evaluación Educativa. La Habana. Cuba.

MARTíNEZ, N. (1997). Autoevaluación Departamental. Anales de Pedagogía. Núm. 12. Murcia. España.

MENGUZZATO, Martina; RENAU, Juan José. (2000). La dirección estratégica de la empresa. Un enfoque innovador del management. Dirección de Capacitación de Cuadros y Estudios de Dirección. MES. Combinado del Libro “Alfredo López. La Habana. Cuba.

MORALES I; FERNÁNDEZ, B. (2009). La evaluación institucional en los centros de educación médica de Cuba. Revista de Educación Médica Superior. Vol. 38. No. 150. La Habana. Cuba.

RONDA, Guillermo. (2007). Dirección estratégica, constructo y dimensiones. Comisión Nacional de Telecomunicaciones, Ediciones Futuro. Republica Bolivariana de Venezuela.

RODRÍGUEZ, Fermín O.; ALEMAÑY, Sonia. (1998). Enfoque, dirección y planificación estratégica. Conceptos y metodología. En: Dirección de Capacitación de Cuadros y Estudios de Dirección. Dirección por objetivos y dirección estratégica. La Experiencia cubana. Compendio de artículos. Editado por el Ministerio de Educación Superior. La Habana. Cuba.

SAMOILOVICH, D. (2008). Tendencias de la educación superior en América Latina. En: Colectivo de autores. CRES- 2008. Capítulo 9. Senderos de innovación. Repensando al gobierno de las universidades públicas de América Latina. Colombia.

UNESCO-IESALC (2008): Declaración Final de la Conferencia Regional de la Educación Superior para América Latina y el Caribe. CRES 2008. Colombia. 